\title{
Celiac disease: a disease with many faces
}

\author{
Riccardo Pratesi*, Lenora Gandolfi*
}

$I_{n}$ study focusing on the significant increase in the prevalence of celiac disease (CD) among Down Syndrome (DS) patients. This increase is comparable with increases found by studies in other countries. ${ }^{1}$ The $5.6 \%$ prevalence observed by the study is within the range of the many different prevalence rates cited in the world literature. The number of patients who the study discovered suffer concomitantly from $C D$ becomes even more significant if we consider that the fifth patient who had a positive antiendomysium antibody (EMAIgA) test almost certainly had celiac disease too. The EMA-IgA test has exhibited high levels of sensitivity and specificity ${ }^{2}$ and, although it does not completely rule out false negative results, in the experience accumulated at our laboratory, no patient with a positive EMA-IgA test ever failed to have the diagnosis confirmed by biopsy.

This is the first work to be done in Brazil on this relationship and the interest that it arouses extrapolates its importance to the care of DS patients. The importance of the article does not arise simply from the confirmation of the existence of a common association between DS and CD. After all, it has long been known that patients with DS and also those with two other genetic syndromes, Turner ${ }^{3}$ and Williams ${ }^{4}$ syndromes, are group at risk of concomitantly presenting CD. Its main value, in our view, is that it calls attention to this clinical entity that is little thought of and, consequently, rarely diagnosed in our country.

We believe that doubts no longer exist that $C D$ is a more common condition in our country than had previously been supposed and one which, as is the case in other parts of the world, can go undiagnosed for prolonged periods. Studies indicate that the time taken to reach a definitive diagnosis of $C D$ can be extremely prolonged and can take over ten

\footnotetext{
* Post-PhD, Università degli Studi, Ancona, Itália. Researcher, Center for Research, Diagnosis and Prevention of the Celiac Disease. Professor, Postgraduation Programs on Medical Sciences and Health Sciences, Universidade de Brasília (UNB), Brasília, DF, Brazil.
}

Suggested citation: Pratesi R, Gandolfi L. Celiac disease: a disease with many faces. J Pediatr (Rio J). 2005;81:357-8. years. ${ }^{5}$ This fact is an example of unnecessary delay before arriving at a diagnosis. The patients described in the study in question can be considered an example of excessive delay before reaching a definitive $C D$ diagnosis. Even though DS patients are known to be an at-risk group for coexisting $C D$ and despite the five EMA-IgA-positive patients being aged from 6 to 18 years, none of them had been previously diagnosed as a carrier of the disease.

Celiac prevalence in European populations or populations with European ancestors varies from 0.3 to $1 \%$ with many cases probably remaining undiagnosed for prolonged periods of time. ${ }^{6}$ In Brazil the available prevalence data are still scarce, but it is probable that the prevalence in our country is not far from the prevalence rates found in the old world. In a first attempt at CD case tracking in Brazil, Gandolfi et al. ${ }^{7}$ found a prevalence of $1: 681(0.14 \%)$ among blood donors, but this prevalence probably does not correspond to the real prevalence in the Brazilian population since the majority of blood donors are men and are presumably healthy without anemia (it is known that CD is more common among women, at a rate of $2: 1$ and that anemia is one of its most common symptoms). In a more recent prevalence study, which had the bias of being carried out with a group of patients using the clinical analysis laboratory at a general hospital, the prevalence observed in children was $1: 185(0.54 \%)$ despite patients with gastrointestinal conditions having been excluded. ${ }^{8}$ These studies lead to the belief that, if the prevalence of $C D$ in Brazil is not the same as that in Europe, it is very close.

Celiac disease can be considered a public health problem worldwide, primarily due to its elevated prevalence, its frequent associations with variable and non-specific morbidity and, over the long term, due to the increased probability that severe complications will appear, primarily osteoporosis and malignant diseases of the gastrointestinal tract.

Celiac disease is an autoimmune condition that can potentially affect any organ and not just the gastrointestinal tract as was previously supposed. Its clinical onset and the appearance of the first symptoms can take place at any age. As they properly pointed out in the study in question, the classical form of the disease, with symptomology directly attributable to malabsorption is nowadays observed in a minority of patients. The wide range of possible symptoms 
varies from individual to individual and even for the same individual during different phases of the disease, making diagnosis even more difficult. Untreated CD often manifests monosymptomatically, as refractive anemia, dermatitis herpetiformis, which can be considered as the dermatolological expression of the disease, delayed menarche and early menopause, infertility, repeated miscarriages, hypertransaminemia, depression, progressive neurological symptomology, primarily ataxia and epilepsy associated with cerebral calcifications, osteoporosis and hypoplasia of dental enamel. Before diagnosis a significant proportion of patients, particularly those identified by tracking studies, described an indefinite general unwellness which they accept as their normal condition, exhibiting clear improvement after the institution of a gluten-free diet.

The growing importance of $C D$ as a public health problem, the inherent difficulties in diagnosing it, very often made more difficult by the diversity and non-specific nature of its clinical expression very often beyond the gastrointestinal system, its progressive severity if not diagnosed in time and the existence of effective treatment by means of a glutenfree diet all raise the controversial issue of systematic screening of the general population. ${ }^{9}$ The primary argument offered by those who advocate this measure is the potential of $C D$ to result in severe complications and that the prevalence of $C D$ far exceeds the prevalence of other diseases for which there are already routine screening programs, as is the case with congenital hypothyroidism, phenylketonuria and congenital hearing deficiency.

Such a measure is obviously inadvisable and even impossible to execute in our country, not just because of the resources required, but also because negative serological tests in individuals genetically predisposed do not rule out the possibility of the disease appearing in the future. A sign of this is that the authors of the study most properly recommend observation and the repetition of serological tests every 2 years for their DS patients, due to the possibility of future $C D$ clinical onset.

In conclusion, for which risk groups is the systematic application of serological tests indicated? Obviously the tests are obligatory for adults and children with the classical form of the disease, primarily characterized by chronic diarrhea, flatulence, abdominal distension and weight loss. A second important risk group, for whom both tests and alert observation are indicated, are the relatives of celiac patients Among these the prevalence of $C D$ can reach $18 \% .{ }^{10}$ Serological tests are also necessary for the genetic syndromes already mentioned: DS and the Turner syndrome and the Williams syndrome. They are also indicated for patients with other autoimmune diseases, in particular type I diabetes, Sjogren syndrome and thyroiditis. They are indicated for patients with neurological abnormalities of indefinite diagnoses, particularly for difficult to control ataxia and epilepsy associated with cerebral calcifications. They are indicated for patients with osteopenia/osteoporosis and sufferers from dermatitis herpetiformis. They are advisable, depending on clinical criteria, for frequently monosymptomatic patients who present non-classic symptoms such as joint pain, frequent apthous stomatitis, chronic constipation, dental enamel defects, chronic hepatitis or persistent hypertransanminemia, refractive iron deficiency anemia, late menarche or early menopause, infertility with no obvious cause and short stature.

In conclusion, the diagnosis of $C D$ primarily depends on thinking of $C D$ in the first place and particularly so in the absence of the classic clinical status since, nowadays, the most often encountered forms are atypical, monosymptomatic or even silent.

\section{References}

1. Nisihara RM, Kotze LM, Utiyama SR, Oliveira NP, Fiedler PT, Messias-Reason IT. Celiac disease in children and adolescents with Down syndrome. J. Pediatr (Rio J). 2005;81:373-6.

2. Hill ID. What are the sensitivity and specificity of serologic tests for celiac disease? do sensitivity and specificity vary in different populations? Gastroenterology. 2005;128:S25-32.

3. Ivarsson SA, Carlsson A, Bredberg A, Alm J, Aronsson S, Gustafsson J, et al. Prevalence of coeliac disease in Turner syndrome. Acta Paediatr. 1999;88:933-6.

4. Giannotti A, Tiberio G, Castro M, Virgilii F, Colistro F, Ferretti F, et al. Coeliac disease in Williams syndrome. J Med Genet. 2001;38:767-8.

5. Fasano A, Berti M, Gerarduzzi T, Not T, Colletti RB, Drago S, et al. Prevalence of celiac disease in at-risk and not-at-risk groups in the United States. Arch Intern Med. 2003;163:286-92.

6. Catassi C, Fornaroli F, Fasano A. Celiac disease: from basic immunology to bedside practice. Clin Appl Immunol Rev. 2002;3:61-71.

7. Gandolfi L, Pratesi R, Cordoba JC, Tauil PL, Gasparin M, Catassi C. Prevalence of celiac disease among blood donors in Brazil. Am J Gastroenterol. 2000;95:689-92.

8. Pratesi R, Gandolfi L, Garcia SG, Modelli IC, Lopes de Almeida $P$, et al. Prevalence of coeliac disease: unexplained age-related variation in the same population. Scand J Gastroenterol. 2003;38:747-50.

9. Mearin ML, Ivarsson A, Dickey W. Coeliac disease: is it time for mass screening? Best Pract Res Clin Gastroenterol. 2005;19: 441-52.

10. Bonamico $M$, Mariani $P$, Mazzilli $M C$, Triglione $P$, Lionetti $P$, Ferrante $P$, et al. Frequency and clinical pattern of celiac disease among siblings of celiac children. J Pediatr Gastroenterol Nutr. 1996;23:159-63. 